\title{
Derivation of the Rotational Frequency of Massive Stars from Seismic Studies
}

\author{
C. Aerts \\ Institute of Astronomy, Celestijnenlaan 200B, B-3001 Leuven, Belgium \\ R. Scuflaire and A. Thoul \\ Institute of Astrophysics and Geophysics, 17 Allée du 6 Août, B-4000 \\ Liège, Belgium
}

\begin{abstract}
In this contribution we review the current status of the determination of the rotational frequency in non-radially pulsating B stars, i.e. $\beta$ Cep stars and slowly pulsating $\mathrm{B}$ stars. Considerable progress is currently being made in the understanding of the non-radial oscillations of main-sequence $\mathrm{B}$ stars by means of high-temporal, high-spatial resolution spectroscopic time series. This has led to the detection of frequency multiplets, which are interpreted as rotationally splitted non-radial modes and which allow an accurate determination of the surface rotational frequency in some stars. We outline how our future goal, i.e. the derivation of the internal rotation frequency, can be achieved.
\end{abstract}

\section{Our Motivation, Goal and Approach}

Massive stars play a crucial role in astrophysics. They are mainly responsible for the synthesis of the heavy elements and so determine the chemical evolution of galaxies. With this in mind, we set ourselves as primary goal to understand in detail the structure of such stars. More particularly, we want to understand the internal rotation of massive stars, as well as the role of convective overshooting and rotational mixing on their evolution. With this knowledge we will be able to understand much better the preamble of supernovae explosions.

The approach we take to achieve our goals is to perform seismic studies of massive main-sequence stars. Two such classes of stars, for which stellar oscillations have been firmly established, are known: the $\beta$ Cep stars and the slowly pulsating B stars. For a recent review on the characteristic physical and pulsational parameters of such stars we refer to De Cat (2002).

For seismic analyses of stars to be successful, one needs to perform several steps. First of all, accurate frequency determination of as many oscillation modes as possible have to be derived. This can be achieved from time series analysis of either high-precision photometric data or line-profile variations derived from high-resolution spectra. Such type of observations are available for many of the $\beta$ Cep stars by now (Sterken \& Jerzykiewicz, 1993; Aerts \& De Cat, 2003 and references therein) and recently also for some 15 slowly pulsating B stars (Aerts et al., 1999; De Cat \& Aerts, 2002).

The second step in a seismic analysis consists of identifying the detected oscillation modes, i.e. to derived their wavenumbers $(\ell, m, n)$ (for the meaning 
of these symbols, see Matthews; Christensen-Dalsgaard - these proceedings). If a sufficient amount of frequencies has been found, one may hope to achieve mode identification from pattern recognition of the frequency spectra. However, for stars in which only a limited amount (typically less than 10) of modes is detected, one needs other methods to disentangle the wavenumbers from the data. An estimate of the degree $\ell$ of the modes can be derived from multicolour photometry (see Dupret et al. 2003 for a recent new version of this technique). Since the introduction of high-resolution spectrographs, however, it has become clear that line-profile variations offer much more detailed diagnostics that allow one to derive not only $(\ell, m)$, but also the amplitudes of the modes and the projected rotation velocity to a high precision (see e.g. Aerts et al., 1992; Telting et al., 1997).

Finally, after frequency detection and mode identification, one may hope to derive information on internal rotation and convective overshooting. For the rotational aspect, on which we will focus here, one tries to make use of the rotational splitting of the oscillation modes through the equation

$$
\sigma_{n \ell m}-\sigma_{n \ell 0}=-m \int_{0}^{R} \Omega(r) K_{n \ell}(r) d r+\Theta\left(\Omega^{2}\right)
$$

in order to derive the internal rotation law $\Omega(r)$ (we neglect differential rotation). In this equation, the kernels $K_{n \ell}$ are functions of the eigenfunctions and the internal structure of the star. This method was used very successfully for the derivation of the internal rotation of the Sun (see Christensen-Dalsgaard - these proceedings). In order to derive $\Omega(r)$ from Eq. (1), it is needed to observe several unequally-spaced frequency multiplets. This has so far not yet been achieved for main-sequence stars more massive than the Sun.

\section{Observational Status for Massive Stars}

The amount of detected frequencies in $\beta$ Cep stars and slowly pulsating B stars from ground-based data is below ten. Moreover, frequency multiplets have seldom been established in these two types of oscillators. One frequency multiplet does not allow to derive $\Omega(r)$, but merely leads to a mean value of the rotation frequency.

\section{1. $\quad \nu$ Eri}

The first $\beta$ Cep star in which a frequency multiplet was firmly established from photometric data is $\nu$ Eri (Kubiak 1980). This triplet, however, has a large departure from equidistance and the second-order rotational correction term $\Theta\left(\Omega^{2}\right)$ indicated symbolically in Eq. (1) is insufficient to explain this, unless the star is seen almost pole-on. This star deserves to be studied in much more detail, as Aerts et al. (1994) clearly have shown it to have considerable lineprofile variations. A large photometric and spectroscopic multisite campaign was therefore recently set up by G. Handler and C. Aerts. It is running at the time of this symposium. Its time base is 3 months for the high-resolution spectroscopy and 5 months for the photometry. This campaign, which will end in April 2003, will tell us what the depth of seismic analyses from the ground for massive stars is and will direct any further efforts in this topic. 

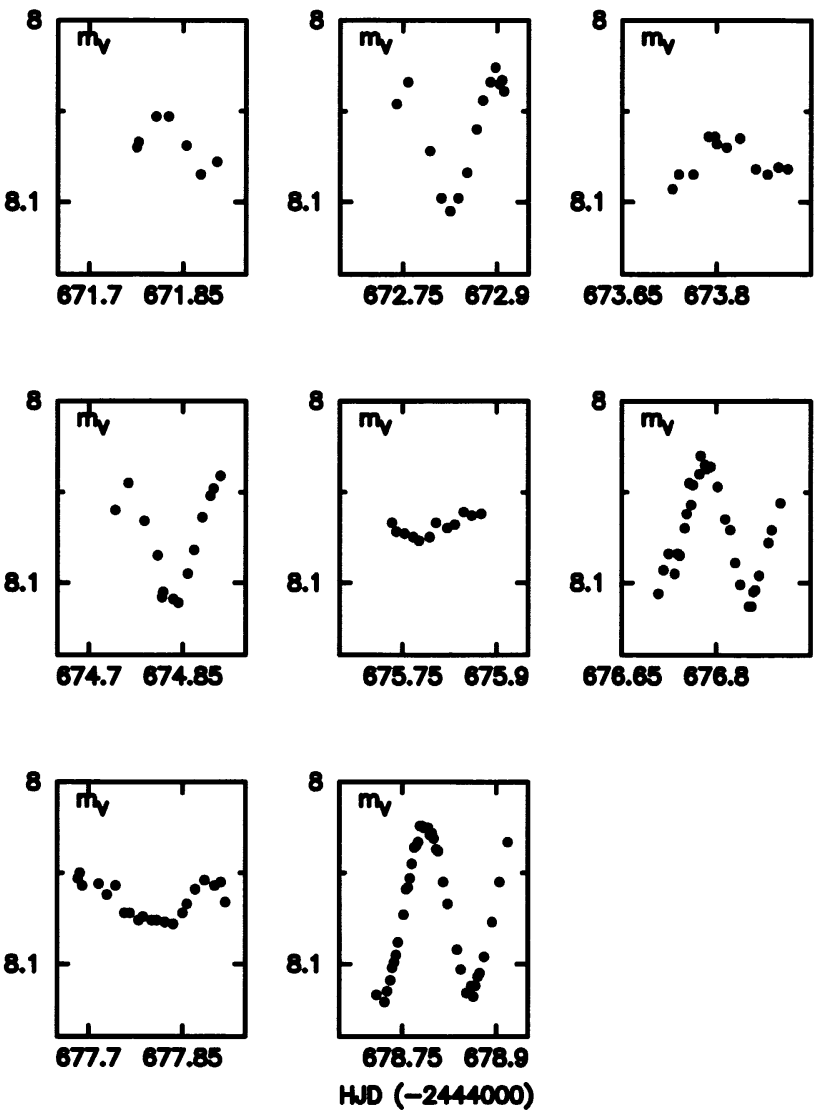

Figure 1. Lightcurve of HD 129929 in the Geneva V band during 8 consecutive nights. 


\section{2. $\beta$ Cep}

One frequency quintuplet has been found by Telting et al. (1997) in the prototype of the $\beta$ Cep stars. From the observed splitting constant one derives $\Omega=0.165 \mathrm{~cd}^{-1}$, i.e. a rotational period of about 6 days. However, UV spectra of the star reveal EW changes with a period of 12 days. A magnetic field was established in $\beta$ Cep (Donati et al., 2002) and Shibahashi \& Aerts (2000) have shown an oblique pulsator model to be compatible with the line-profile variations for a rotational period of 6 days. This oblique pulsator model, however, as well as the observational accuracy of the quintuplet frequencies, need refinement before it can lead to more detailed information on the internal rotation.

\subsection{HD 129929}

A new interesting case of multiplet detection we report here occurs for the highgalactic latitude $\beta$ Cep star HD 129929. We have gathered multicolour Geneva photometry for this star during 1978 - 1997. From the 1493 data points we derive at least six frequencies. Three of these were reported earlier by Waelkens \& Rufener (1983) while Heynderickx (1992) also established three frequencies of which two are in common to those found by Waelkens \& Rufener.

The multiperiodicity of this star becomes evident from the lightcurves obtained during 8 consecutive nights shown in Fig. 1 for the visual Geneva data. The addition of a significant amount of data since the study by Heynderickx (1992) has allowed us to refine the frequency analysis considerably and we have now firmly establised the following five frequencies in the star: $6.4375,6.4496$, $6.4617,6.9783,6.9904,7.5904 \mathrm{~cd}^{-1}$. An additional frequency peak occurs near $6.9662 \mathrm{c} \mathrm{d}^{-1}$. It is immediately evident from these frequency values that we are dealing with (parts of) two multiplets, of which the separation amounts to $\Omega=0.0121 \mathrm{~cd}^{-1}$. Photometric mode identification will be done in the near future, but in any case HD 129929 is the first pulsating B star in which more than one multiplet has been found.

For completeness we mention that we are running extensive observational programmes to gather multicolour photometry and high-resolution spectroscopy of slowly pulsating B stars. The gravity-modes in these stars, however, have periods of typically a few days, leading to beat-periods of several months. Our data reveal so far several frequencies (De Cat \& Aerts 2002) but are insufficient in amount and time spread to establish firmly the presence of multiplets in these gravity-mode B-type pulsators.

\section{A Detailed Case Study: EN Lacertae}

A slightly different type of approach to derive $\Omega$ can be followed when accurate frequency values and accompanying unambiguous mode identifications are achieved for several oscillation modes. Such a situation occurs for the $\beta$ Cep star EN (16) Lac, for which the following three frequencies are well established: $f_{1}=5.9113 \mathrm{~cd}^{-1}, f_{2}=5.8529 \mathrm{~cd}^{-1}, f_{3}=5.5026 \mathrm{~cd}^{-1}$ (Lehmann et al., 2001). Aerts et al. (2003) performed for the first time spectroscopic mode identification in this star and found the two modes with close frequencies $f_{1}$ and $f_{2}$ to be axisymmetric, with $\ell=0$ and with $\ell=2$ respectively. The identification of the 
third mode is $\ell=1$. Overall agreement was found with the photometric mode identification of these three well-established frequencies of the star.

The results of this mode identification were used as starting point for seismic modelling, starting from the known stellar parameters (the star is a member of an eclipsing binary). This has allowed us to derive a small region of acceptable mass and metallicity of the star, to unprecedented precision for a B-type star (Aerts et al., in preparation). A by-product of this modelling is that the three possibilities for the third mode, $\ell=1$ and $m=-1,0,1$, lead to a value for $\Omega(R)$ in the two cases $m= \pm 1$. While there still is some ambiguity left for the rotational period of this star, it does serve as a good example of how seismic information can be used to study stellar rotation in massive stars. Should we be able to detect a larger number of modes, and identify some of them as $m=0$, then the frequency values of the $m \neq 0$ modes allows the determination of $\Omega(r)$ through Eq. (1) provided that the stellar models are sufficiently accurate to reproduce well the axisymmetric modes.

\section{Future Prospects}

It is clear that additional spectroscopic multisite campaigns will be set up in the near future should our current campaign on $\nu$ Eri turn out to be sufficient to allow the derivation of (constraints on) the (internal) rotation frequency of that $\beta$ Cep star. We expect to be able to report our results of that campaign within about one year from now.

Ideally, one would hope to detect a sufficient amount of modes so that frequency inversion becomes possible. Once at that stage, improvements in the theoretical models so that the predicted frequencies perfectly match the observed ones can be aimed at. We are yet still far away from this level of precision for massive oscillators with $\kappa$-driven modes, the main reason being the limited number of detected modes. The example of WIRE data of the $\beta$ Cep star $\beta$ Crucis (Cuypers et al., 2002) indicates that the future asteroseismic and planet-finding space missions will imply a major step forward in seismic studies of massive stars. In view of their key-role in stellar and galactic evolution we therefore suggest to include several massive oscillators in the target lists of these space missions.

\section{References}

Aerts, C., De Pauw, M., Waelkens, C. 1992, A\&A 266, 294

Aerts, C., De Cat, P. 2003, SSRv, in press

Aerts, C., De Cat, P., Peeters, E., et al. 1999, A\&A 343, 872

Aerts, C., Lehmann, H., Briquet, M., et al. 2003, A\&A, in press

Aerts, C., Waelkens, C., De Pauw, M. 1994, A\&A 286, 136

Cuypers, J., Aerts, C., Buzasi, D., et al. 2002, A\&A 392, 599

De Cat, P. 2002, In C. Aerts, T.R. Bedding \& J. Christensen-Dalsgaard (eds.), Radial and non-radial pulsations as probes of stellar physics, ASP Conf. Ser., Vol. 259, 196 (San Francisco: ASP)

De Cat, P., Aerts, C. 2002, A\&A 393, 965 
Heynderickx, D. 1992, A\&AS 96, 207

Kubiak, M. 1980, Acta Astron. 30, 219

Lehmann, H., Harmanec, P., Aerts, C., et al. 2001, A\&A 367, 236

Shibahashi, H., Aerts, C., 2000, ApJ, L143

Sterken, C., Jerzykiewicz, M., 1993, SSRv 62, 95

Telting, J.H., Aerts, C., Mathias, P. 1997, A\&A 322, 493

Waelkens, C., Rufener, F. 1983, A\&A 119, 279 